\title{
AMENABLE RELATIONS FOR ENDOMORPHISMS
}

\author{
JANE M. HAWKINS
}

\begin{abstract}
We give necessary and sufficient conditions for an endomorphism to admit an equivalent invariant $\sigma$-finite measure in terms of a generalized PerronFrobenius operator. The assumptions are that the endomorphism is nonsingular (preserves sets of measure zero), conservative, and finite-to-1. We study two orbit equivalence relations associated to an endomorphism, and their connections to nonsingularity, ergodicity, and exactness. We also discuss Radon-Nikodym derivative cocycles for the relations and the endomorphism, and relate these to the Jacobian of the endomorphism.
\end{abstract}

\section{INTRODUCTION}

To every ergodic nonsingular automorphism of a Lebesgue space one can associate amenable equivalence relations in several natural ways. This paper continues a study begun in [10] on invariants for endomorphism under a type of orbit equivalence which is weaker than isomorphism but much stronger than the analogous concept in the invertible case. We also consider Radon-Nikodym derivatives for the relation as well as the Radon-Nikodym cocycle for an endormorphism. In the invertible case, whether one considers the Jacobian function for a transformation, the Radon-Nikodym derivative of the mapping or the Radon-Nikodym derivative of the associated amenable equivalence relation, it is the same cocycle and purely a question of notation. The situation is completely different in the noninvertible case (see for example, $[7,10-12])$. This paper contains in addition to a discussion about the various cocycles mentioned above, some attempts to draw connections between these objects. There are still many open question in this area, including what the best notion of weak orbit equivalence for noninvertible maps is and its invariants.

In $\S 1$ we introduce the relation $R_{T}$ and a subrelation $S_{T}$ associated to any endomorphism. $T$. The main results proved in this section are that $R_{T}$ is nonsingular and ergodic if and only if $T$ is (nonsingular means both forward and backward nonsingular); if $T$ is nonsingular, then $S_{T}$ is nonsingular but the converse is false. If $T$ is nonsingular, then $S_{T}$ is ergodic if and only if $T$ is exact. Some of the results in $\S 1$ for finite measure-preserving endomorphisms are implicit in a paper of Rohlin [18], and some have appeared recently in [21]. In $\S 2$ we also use conditions of Kakutani [13] to give examples of invertible odometers giving rise to ergodic nonsingular and singular one-sided shifts on

Received by the editors July 22, 1991 and, in revised form, May 12, 1992.

1991 Mathematics Subject Classification. Primary 28D99, 28D05, 58F11. 
the same measure space. Section 3 is a comparison between the Jacobian function which measures the local measure-theoretic properties of an endomorphism $T$ and the Radon-Nikodym derivative for the relations $S_{T}$ and $R_{T}$. In $\S 4$ we discuss the Radon-Nikodym derivative cocycle $\omega_{\mu}$ for the endomorphism, a cocycle for the semigroup action generated by $T$. We give necessary and sufficient conditions on $\omega_{\mu}$ for $T$ to admit a $\sigma$-finite invariant measure equivalent to $\mu$, and in $\S 5$ we study recurrence of measures and Markovians. Section 6 contains some generalizations to countable-to- 1 endomorphisms, including a short discussion of an example of Schmidt [21]. We conclude the paper with a section connecting the relations $R$ and $S$ to $\omega_{\mu}$ and mention a few open problems.

The author would like to thank Karma Dajani, Stanley Eigen, Klaus Schmidt, and Cesar Silva for useful discussions concerning this paper, and Bruce Kitchens for his hospitality at IBM in Yorktown Heights where an early draft of this paper was written in 1988 . We also thank the referee for many useful comments.

\section{Properties of the RElations $R$ AND $S$ FOR AN ENDOMORPHISM}

We begin by defining two relations of interest to us in this paper; one is a subrelation of the other. Throughout we assume that $T$ is a nonsingular endomorphism of a Lebesgue space $(X, \mathscr{B}, \mu)$, with $\mu$ a $\sigma$-finite measure; in addition we assume that $T$ is at most countable-to-1. By a result of Rohlin [17] we can assume by replacing $X$ by a measurable $T$-invariant subset of full measure if necessary that $T$ is forward nonsingular as well so that $T$ satisfies: for all $A \in \mathscr{B}, \mu(A)=0 \Leftrightarrow \mu\left(T^{-1} A\right)=0 \Leftrightarrow \mu(T A)=0$. We will therefore have a standing assumption throughout this paper that $T$ nonsingular means $T$ is both forward and backward nonsingular. It is well known that there exists a partition of $X$ into at most countably many pieces $\zeta=\left\{A_{1}, A_{2}, A_{3}, \ldots\right\}$ such that $\mu\left(A_{i}\right)>0$ and the restriction of $T$ to each $A_{i}$, which we will write as $T_{i}$, is one-to-one. In particular, let $\varepsilon$ denote the point partition of $X$; we choose each $A_{i}$ to be a maximal one-sheeted set of $T^{-1} \varepsilon$ [17], and we number the atoms so that $\mu\left(T A_{i}\right) \geq \mu\left(T A_{i+1}\right)$ for each $i$. We can assume then, since $T$ is surjective, that $T_{1}$ is one-to-one and onto $X$. We will call such a partition a Rohlin partition for $T$. (It is not unique.) We define $T$ to be $n$-to- 1 if every Rohlin partition $\zeta=\left\{A_{1}, A_{2}, A_{3}, \ldots\right\}$ contains precisely $n$ atoms with $T_{i}$ one-to-one and onto $X$ for each $i=1, \ldots, n$. Equivalently, for $\mu$-a.e. $x \in X$, the set $T^{-1} x$ contains exactly $n$ points. We call $T$ bounded-to- 1 if every Rohlin partition has only finitely many atoms. (The number of atoms will be independent of the choice of $\zeta$.) For each fixed Rohlin partition $\zeta$, a unique partition $\zeta_{k}$ is uniquely determined up to labelling for the endomorphism $T^{k}$ for all $k>1$; it is given by $\zeta_{k}=\bigvee_{i=0}^{k-1} T^{-i} \zeta$.

We define an equivalence relation on $X \times X$ as follows.

Definition 1.1. (1) The point $(x, y) \in X \times X$ is in $R_{T}$ if and only if $T^{n} x=T^{m} y$ for some $n, m \geq 1$.

(2) We say $(x, y) \in S_{T} \subseteq R_{T}$ if $T^{n} x=T^{n} y$ for some $n \geq 1$.

It is well known (cf. [4]) that $R_{T}$ and $S_{T}$ are amenable measurable equivalence relations on $X$, and such relations are classified up to isomorphism [14, 4]. When it is clearly understood that the transformation under study is $T$, 
we simply write $R$ and $S$ for $R_{T}$ and $S_{T}$ respectively. We remark that $T$ is invertible if and only if $S$ is trivial (each equivalence class consists of exactly one point). In this case, $R(x) \equiv\{y:(x, y) \in R\} \in\left\{T^{n} x\right\}_{n \in \mathbb{Z}}$.

As in [10], we define two endomorphisms $T$ and $T^{\prime}$ to be orbit equivalent if $R_{T}$ and $R_{T^{\prime}}$ are isomorphic. We say $T$ and $T^{\prime}$ are strongly orbit equivalent if $R_{T} \simeq R_{T^{\prime}}$ and the isomorphism $\psi$ implementing the orbit equivalence satisfies $\psi \times \psi\left(S_{T}\right)=S_{T^{\prime}}$. The $\left(\frac{1}{2}, \frac{1}{2}\right)$ i.i.d. Bernoulli shift and the $\left(\frac{1}{4}, \frac{1}{4}, \frac{1}{2}\right)$ i.i.d. Bernoulli shift are orbit equivalent but not strongly orbit equivalent [10]. Since nonsingular ergodic countable amenable equivalence relations have been completely classified up to isomorphism [14,4], it is interesting to study $S_{T}$ and $R_{T}$ for an endomorphism $T$ to see how much information they carry about the original endomorphism. We recall some basic terminology about these relations. These relations are also discussed in [21].

Definition 1.2. Assume that $R=R_{T}$ is the measurable relation defined above for $T$.

(1) For every set $A \in \mathscr{B}$,

$$
R(A)=\{y \in X:(x, y) \in R \text { for some } x \in A\}=\bigcup_{x \in A} R(x),
$$

where $R(x)$ is the equivalence class of $x$.

(2) We say that the equivalence relation $R$ is nonsingular (w.r.t. $\mu$ on $X$ ) if $\mu(A)=0 \Leftrightarrow \mu(R(A))=0$ for all $A \in \mathscr{B}$.

(3) $R$ is ergodic (w.r.t. $\mu$ ) if $R(A)=A \Rightarrow \mu(A)=0$ or $\mu(X \backslash A)=0$.

By $\operatorname{Aut}(X)$ we mean the set of all measurable, invertible nonsingular mappings (automorphisms) of $X$.

(4) The full group of $R$, is defined by

$$
[R]=\left\{V \in \operatorname{Aut}(X):(x, V x) \in R_{T} \text { for a.e. } x \in X\right\} .
$$

Similarly we define

$$
[S]=\left\{U \in \operatorname{Aut}(X):(x, U x) \in S_{T} \text { for a.e. } x \in X\right\} .
$$

(5) A partial isomorphism of $R$ is an element of the full groupoid of $R$ denoted [[R]]. We define $[[R]]=\{\phi: A \rightarrow B, A, B \in \mathscr{B}, \phi$ is one-to-one, onto $B$, nonsingular, and $(x, \phi x) \in R_{T}$ for all $\left.x \in A\right\}$.

Clearly $[R] \subseteq[[R]]$ and $[S] \subseteq[[S]] \subseteq[[R]]$. We remark that if $V \in[[S]]$, $V$ must have the property that for $\mu$ a.e. $x, T^{n(x)} x=T^{n(x)}(V x)$ for some $n(x) \in \mathbb{N}$. Therefore $V x \in\left\{T^{-n}\left(T^{n} x\right)\right\}_{n \in \mathbb{N}}$; if $x \neq V x$, then $x$ and $V x$ lie in different atoms of the partition $\zeta_{n}$ corresponding to $T^{n}$.

There are obvious connections between the ergodicity and nonsingularity of $T$ and that of $R_{T}$ and $S_{T}$; in particular, we show in the lemma below that they are the same for the case of $T$ and $R_{T}$. The connections between $T$ and $S_{T}$ are more interesting, but we begin by proving that the ergodicity and nonsingularity of $T$ and $R_{T}$ are equivalent notions.

Lemma 1.3. (1) $T$ is nonsingular $\Leftrightarrow R_{T}$ is nonsingular.

(2) $T$ is ergodic $\Leftrightarrow R_{T}$ is ergodic.

Proof of $(1)$. $(\Leftrightarrow)$ If $T$ is singular, then there exists $A \in \mathscr{B}, \mu(A)>0$ such that either (a) $\mu\left(T^{-1} A\right)=0$, or (b) $\mu(T A)=0$. If (a) occurs then let $B=T^{-1} A$, so $\mu(B)=0$, but $\mu(R(B)) \geq \mu(A)>0$. Therefore $R$ is singular. Case (b) is proved similarly. 
$(\Rightarrow)$ If $R$ is singular, then there exists a set $A, \mu(A)=0$, but $\mu(R(A))>0$. This means there exists a partial isomorphism $f: A \rightarrow B$ such that $\mu(A)=0$ but $\mu(B)>0$, with $B=f A$ and $(A, f A) \in R_{T}$. Therefore there exists a set $C \subseteq B, \mu(C)>0$ and fixed $m, n \in \mathbb{N}$ such that $f^{-1} y=T_{i}^{-n} T^{m} y$ for all $y \in C$. Since $\mu\left(f^{-1} C\right)=0$, it follows that $T$ is singular too.

Proof of (2). It suffices to show that for $A \in \mathscr{B}, T^{-1} A=A$ if and only if $R(A)=A$. From definition 1.1(2) we have that for any $x$,

$$
R(x)=\left\{T^{-m}\left(T^{n} x\right)\right\}_{m, n \geq 1}=R\left(T^{-1} x\right)=T^{-1}(R(x)) .
$$

Then $R(A)=R\left(T^{-1} A\right)=T^{-1}(R(A))$ for $A \in \mathscr{B}$, and the result follows immediately.

We now turn to the connections between $T$ and the subrelation $S_{T}$. First, nonsingularity of $T$ implies nonsingularity of $S_{T}$, even though the converse is not true as was shown in [10] and Example 2.1 below. Second, ergodicity of $S_{T}$ forces the ergodicity of $T$ when the measure is nonsingular for both $S_{T}$ and $T$. This is obvious, but the converse is not true. The ergodicity of $S_{T}$ forces the ergodicity of $R_{T}$ which, as shown above is equivalent to the ergodicity of $T$. But more precisely, ergodicity of $S_{T}$ is equivalent to exactness of $T$, which is proved in Theorem 1.6.

Lemma 1.4. For any set $A \in \mathscr{B}$,

$$
S(A)=A \cup T^{-1}(T A) \cup T^{-2}\left(T^{2} A\right) \cup \cdots=\bigcup_{n=0}^{\infty} T^{-n}\left(T^{n} A\right) \quad(\mu \bmod 0) .
$$

Proof. Since $S(x)=\left\{T^{-n}\left(T^{n} x\right)\right\}_{n \in \mathbb{N}}$, the result follows immediately.

Lemma 1.5. If $T$ is nonsingular, then $S$ is nonsingular.

Proof. Suppose $S$ is singular; then there exists a set $A \subset X$ such that $\mu(A)=0$ but $\mu(S(A))>0$. By Lemma 1.4, $S(A)=\bigcup_{n=1}^{\infty} T^{-n}\left(T^{n} A\right)$. Now $\mu(A)=$ $0 \Rightarrow$ for $n \in \mathbb{N}, \mu\left(T^{n} A\right)=0$ by the nonsingularity of $T$; this implies that $\mu\left(T^{-n}\left(T^{n} A\right)\right)=0$ but this contradicts the above.

We recall that a nonsingular endomorphism $T$ is exact if $\bigcap_{n=0}^{\infty} T^{-n} \mathscr{B}=$ $\{X, \varnothing\} \quad(\mu \bmod 0)$; that is, if $A \in \bigcap_{n=0}^{\infty} T^{-n} \mathscr{B}$, then $\mu(A)=0$ or $\mu(X \backslash A)=$ 0 . We show here that ergodicity of $S_{T}$ is equivalent to exactness of $T$ for $T$ any nonsingular endomorphism. This result in the measure-preserving case is implicit in a paper of Rohlin [18].

Theorem 1.6. If $T$ is a nonsingular endomorphism of a probability space, then $S_{T}$ is ergodic if and only if $T$ is exact.

Proof. Consider any set $A \in \bigcap_{n=0}^{\infty} T^{-n \mathscr{B}}$; then we can write $A=T^{-n}\left(T^{n} A\right)$ $(\mu \bmod 0)$ for all $n \geq 1$. Therefore $S(A)=\bigcup_{n=1}^{\infty} T^{-n}\left(T^{n} A\right)=\bigcup_{n=1}^{\infty} A=$ $A$. Conversely, if $S(\vec{A})=A$, then $A \in \bigcap_{n=0}^{\infty} T^{-n \mathscr{B}}$; this gives a one-to-one correspondence between sets in $\bigcap_{n=0}^{\infty} T^{-n} \mathscr{B}$ and $S$-invariant sets. Ergodicity of $S$ implies if $S(A)=A$, then $\mu(A)=0$ or $\mu(X \backslash A)=0$, which is equivalent to the exactness of $T$.

We obtain the following corollaries to the above theorem. 
Corollary 1.7. If $T$ is an expanding endomorphism of a closed manifold $M$ with Hölder continuous derivative $D_{x} T$ (in $x$ ), then $R_{T}$ and $S_{T}$ are both ergodic relations with respect to Lebesgue measure.

Corollary 1.8. An expanding map $T$ of a bounded metric space with a Borel probability measure has $R_{T}$ and $S_{T}$ ergodic.

Proofs. In both cases $T$ is exact (cf. [15]).

Example 1.9. We give an example of an ergodic measure-preserving endomorphism $F$ of a Lebesgue probability space for which $S_{F}$ is not ergodic. This is an example of a nonexact endomorphism similar to one given in [12]. The space on which we construct $F$ is $T^{2} \simeq \mathbb{R}^{2} / \mathbb{Z}^{2}$, endowed with the $\sigma$-algebra of Borel sets and Lebesgue measure. We define two maps of the circle, $g(x) \equiv 2 x$ $(\bmod 1)$ and $f(x)=x+\alpha(\bmod 1)$ with $\alpha$ irrational in $(0,1)$. Then on $T^{2}$ we define $F(x, y)=(g(x), f(y))=(2 x(\bmod 1), x+\alpha(\bmod 1))$. Since $g$ is exact and $f$ is finite measure preserving the ergodic, $T$ is ergodic [1]. We produce nontrivial invariant sets for the relation $S_{F}$ as follows. Let $A$ be any set in $T^{1}$ satisfying $0<\mu(A)<1$. Then consider the set $B=T^{1} \times A$; it has Lebesgue measure strictly between 0 and 1 . Furthermore we claim that $B=S(B)=\bigcup_{n=1}^{\infty} F^{-n}\left(F^{n} B\right)$. This is clear by computing that

$$
\begin{aligned}
F^{-n}\left(F^{n} B\right) & =\left\{(x, y): F^{n}(x, y) \in F^{n} B\right\} \\
& =\left\{(x, y):\left(2^{n} x(\bmod 1), y+n \alpha(\bmod 1)\right) \in T^{1} \times f^{n} A\right\} .
\end{aligned}
$$

But $f$ is invertible, so $\left.f^{-n}\left(f^{n} A\right)\right)=A$, and therefore $F^{-n}\left(F^{n} B\right)=B$. This shows that $S_{F}$ is not ergodic; the relation $R_{F}$ is ergodic by Lemma 1.3(2).

\section{MEASURES FOR SHIFTS AND ODOMETERS}

Another connection between $T$ and $S_{T}$ has been studied by the author in [10]. Each isomorphism class of $S$ corresponds to an orbit equivalence class of an odometer, and in fact a binary odometer with some nonsingular ergodic measure [14]. One can realize every isomorphism class of $S$ first as an odometer (where we define two points to be related if and only if they are in the same two-sided orbit under the odometer action), then as the relation $S_{T}$ for a onesided shift $T$. This is discussed and proved in [10]. In [10] an example is given of an ergodic nonsingular (type $\mathrm{II}_{\infty}$ ) relation $S_{T}$ coming from a onesided shift which is singular. That this should happen for many choices of an odometer can be seen by studying the conditions set out by Kakutani in [13] for products measures. One can determine which product odometers on $n$ states are realizable as the relations $S_{T}$ for a nonsingular shift $T$ (on the same space). We briefly review the conditions.

A distance between measures is defined by $\rho\left(m, m^{\prime}\right)=\int_{X} \sqrt{\phi(x)} d m(x)$ for measures $m, m^{\prime}$ on $X$ where $\phi(x)=d m^{\prime}(x) / d m(x)$ is the Radon-Nikodym derivative of $m^{\prime}$ with respect to $m$. Equivalently, one defines the metric

$$
\sigma\left(m, m^{\prime}\right)=-\log \rho\left(m, m^{\prime}\right)=-\log \int_{X} \sqrt{\phi(x)} d m(x) .
$$

If we let $X$ be the infinite product space $X=\prod_{j=0}^{\infty}\{0,1, \ldots, n-1\}_{j}$, and $m=\prod_{j=0}^{\infty} m_{j}, m^{\prime}=\prod_{j=0}^{\infty} m_{j}^{\prime}$, with each $m_{j}$ and $m_{j}^{\prime}$ a probability measure 
on $X_{j}=\{0,1, \ldots, n-1\}$, then Kakutani showed that

$$
m \sim m^{\prime} \Leftrightarrow \prod_{j=0}^{\infty} \rho\left(m_{j}, m_{j}^{\prime}\right)>0 \Leftrightarrow \sum_{j=0}^{\infty} \sigma\left(m_{j}, m_{j}^{\prime}\right)<\infty .
$$

We now consider $m$ to be any ergodic nonsingular product measure for the odometer. To check whether $m$ is also a nonsingular measure for the one-sided shift on $X$, we apply Kakutani's condition to the measures $m$ and $m T^{-1}$. A computation shows that a necessary condition for the product measure to be nonsingular for the shift is that, as $j$ gets large, the measures on each factor, $m_{j}, m_{j+1}, \ldots, m_{j+k}$, need to be getting strictly closer to each other as measures on $\{0,1, \ldots, n-1\}$.

Example 2.1. A simple example of an ergodic nonsingular odometer measure giving rise to a singular shift $T$ is the following. We choose any $\alpha$ and $\beta$ in $(0,1)$ with $\alpha \neq \beta$. Then for all even $n \in \mathbb{N}$, let $\mu_{n}$ be given by the distribution $(\alpha, 1-\alpha)$; for all odd $n \in \mathbb{N}$, let $\mu_{n}$ be given by the distribution $(\beta, 1-\beta)$. It is straightforward to compute that the infinite product measure $\mu$ is ergodic and nonsingular for the binary odometer. It is also easy to compute that this measure does not satisfy the conditions of Kakutani so does not give a nonsingular measure for the shift $T$. Under some additional hypotheses on $\alpha$ and $\beta$, we can control the ratio set of the odometer (i.e., the isomorphism class of the relation $S)$. For example, fixing any $\lambda \in(0,1)$, and choosing $\alpha=\lambda /(1+\lambda)$ and $\beta=1-\alpha$ gives a measure $\mu$ of type III $_{\lambda}$ for the odometer. We obtain a type $\mathrm{III}_{1}$ relation $S$ (or odometer) by choosing $\alpha=\lambda /(1+\lambda)$ and $\beta=\gamma /(1+\gamma)$, with $\log \lambda$ and $\log \gamma$ rationally independent.

These examples show that nonsingularity of $T$ is not inherited from the subrelation $S_{T}$ and that Lemma 1.3(1) is the strongest result possible. Letting $T^{\prime}$ denote the i.i.d. one-sided $(\lambda /(1+\lambda), 1 /(1+\lambda))$ Bernoulli shift, and using $T$ and the III $_{\lambda} \mu$ described above, we have that $S_{T} \simeq S_{T^{\prime}}$. However, $T$ is singular and $T^{\prime}$ is nonsingular, so $R_{T}$ is not isomorphic to $R_{T^{\prime}}$.

Example 2.2. An example given in [10] with the property that the relation $S_{T}$ is nonsingular, ergodic, $\Pi_{\infty}$, but the associated shift $T$ is singular has measure defined as follows: for $n \in \mathbb{N}$,

$$
\mu_{2 n}(0)=\mu_{2 n}(1)=\frac{1}{2}, \quad \mu_{2 n-1}(0)=\frac{1}{(n+1)^{2}}, \quad \mu_{2 n-1}(1)=1-\frac{1}{(n+1)^{2}} .
$$

Example 2.3. The above conditions for the nonsingularity and ergodicity of shifts were explored in papers by Krengel [26] and Hamachi [8]. Hamachi gives an example of a two-sided shift with a product measure for which no equivalent invariant measure exists. By looking at that example as a one-sided shift, it can be shown that the one-sided shift retains the same measure-theoretic properties as its two-sided counterpart; i.e., we obtain an exact shift which admits no equivalent $\sigma$-finite invariant measure.

We obtain the following additional corollaries to Theorem 1.6.

Corollary 2.4. Let $X$ denote the one-sided shift space on $n$ states, and let $\mu$ be an ergodic nonsingular measure for the odometer on $X$. Then $\mu$ is nonsingular 
for the shift $T$ on $X$ if and only if $T$ is nonsingular and exact with respect to $\mu$.

Corollary 2.5. Let $X$ denote the one-sided shift space on $n$ states, and let $\mu$ be an ergodic nonsingular product measure for the shift on $X$. Then $\mu$ is an exact measure for the shift.

Proof. If $\mu$ is a product measure which is nonsingular and ergodic for the shift, then it is ergodic for the odometer (cf. [9]), so it is exact.

We now apply a characterization of Brown and Dooley [2] of ergodic odometer measures to exactness of shifts. We assume as above that

$$
X=\prod_{j=0}^{\infty}\{0,1, \ldots, n-1\}_{j}
$$

(although their result is true for more general odometer spaces), with $\mathscr{B}$ the Borel sets and $\mu$ any measure on $\mathscr{B}$.

Definition 2.6. Let $\mathscr{B}_{j}$ be the sub- $\sigma$-algebra of $\mathscr{B}$ generated by rectangles $\left\{\prod_{k=1}^{j} A_{k} \times \prod_{k=j+1}^{\infty} X_{k}\right.$, with $A_{k}$ a Borel set of $\left.X_{k}\right\}$, and let $\mathscr{B}^{j}$ be the sub$\sigma$-algebra generated by the rectangles $\left\{\prod_{k=1}^{j} X_{k} \times \prod_{k=j+1}^{\infty} A_{k}\right.$, with $A_{k}$ a Borel set of $X_{k}$ and almost every $A_{k}$ is $X_{k}$ \}. The measure $\mu$ is a weak product type if for every $j$ there is $n(j) \geq j$ such that

$$
\mu(A \cap B)=\mu(A) \mu(B) \quad \text { for all } A \in \mathscr{B}_{j}, B \in \mathscr{B}^{n(j)} .
$$

Note: $\mu$ is of product type if $n(j)=j$ works for all $j$. From [2] the following corollary is immediate.

Corollary 2.7. Let $X$ denote the one-sided shift space on $n$ states, and let $\mu$ be an ergodic nonsingular measure for the shift on $X$. Then $\mu$ is exact for the shift if and only if $\mu$ is of weak product type.

\section{THE JACOBIAN FOR $T$ AND THE RADON-NIKODYM DERIVATIVES FOR $R_{T}$ AND $S_{T}$}

We consider some connections between the full group of the relations of an endomorphism and the Jacobian function defined by Parry [16] for an endomorphism. Using the notation of $\S 1$, we choose a Rohlin partition $\zeta=\left\{A_{1}, \ldots\right\}$ for $T$; we note that the measure $\left.\mu T\right|_{A_{i}} \equiv \mu T_{i}$ is absolutely continuous with respect to $\mu$. The Jacobian of $T$ is then defined by

$$
J_{\mu T}(x)=\sum_{i} \chi_{A_{i}}(x) \frac{d \mu T_{i}}{d \mu}(x) .
$$

The Jacobian is independent of the choice of Rohlin partition $\zeta$ [16].

We will first consider endomorphisms whose Rohlin partitions have exactly $n$ atoms. We remark that while most of the results in this section have straightforward proofs if the endomorphism $T$ is a shift on $n$ states, we cannot make that assumption. The reason is that any $n$-to- 1 measure-preserving shift has measure theoretic entropy at most $\log n$. We give two different types of examples of $n$-to-1 endomorphisms whose entropy is greater than $\log n$. 
Examples 3.1. 1. Consider the Cartesian product of any invertible measurepreserving ergodic transformation with infinite entropy and a 1-sided Bernoulli shift on $n$ states. The product map is $n$-to-1, ergodic, and has infinite entropy. This map is not exact.

2. A different example which is exact, and four-to-one on the two-dimensional torus is given by the matrix $A=\left[\begin{array}{ll}4 & 2 \\ 2 & 2\end{array}\right]$ (which induces an endomorphism on $\mathbb{R}^{2} / \mathbb{Z}^{2}$ in the usual way). One computes from [6] that the entropy is too large $(>\log 4)$ for this to be written as a shift on four states.

The results below indicate that nevertheless there is present the structure of a shift (or odometer) on $n$ states in such endomorphisms.

Theorem 3.2. Suppose $T$ is a nonsingular $n$-to-1 endomorphism of $(X, \mathscr{B}, \mu)$. Then there is an automorphism $V \in[S], V \neq I d$, such that $d \mu V / d \mu$ is a coboundary for $V$ with transfer function $J_{\mu T}$. Furthermore $T \circ V=T$ a.e.

Proof. We will define $V \in[S]$ as follows. We choose and fix a Rohlin partition $\zeta$; we will define $V$ to map $A_{1}$ injectively onto $A_{2}, A_{2}$ onto $A_{3}$, etc., and $A_{n}$ onto $A_{1}$. For each $x \in A_{1}$, there is a unique $y \in A_{2}$ such that $T x=T y$. We define $V x=y \in A_{2}$; in particular, $V x=T_{2}^{-1} \circ T_{1}(x)$. We define $V$ in this way on every atom of the partition of $X$ given by $\zeta$; for $x \in A_{k}$, $V x=T_{k+1}^{-1} \circ T_{k}(x)$ if $k \leq n-1$. For $x \in A_{n}, V x=T_{1}^{-1} \circ T_{n}(x)$. We have defined a one-to-one map of $X$ onto itself which preserves the partition $\zeta . V$ is clearly not ergodic with respect to $\mu$; fix any set $B \subseteq A_{1}$ of positive measure, and look at its translates under $V$. The set $B^{\prime}=B \cup V B \cup \cdots \cup V^{n-1} B$ is invariant under $V$. In fact $V$ is periodic and $V^{n}=\mathrm{Id}$. We now compute the Radon-Nikodym derivative of $V$. For $i<n$, if $x \in A_{i}, V x=T_{i+1}^{-1} \circ T_{i}(x)$. Therefore

$$
\frac{d \mu V}{d \mu}(x)=\frac{d \mu T_{i+1}^{-1}}{d \mu}\left(T_{i} x\right) \frac{d \mu T_{i}}{d \mu}(x)=\frac{d \mu T_{i+1}^{-1}}{d \mu}\left(T_{i} x\right) J_{\mu T}(x) .
$$

A computation shows that if $x \in A_{i}, T_{i} x=T x=T V x$ and

$$
\frac{d \mu T_{i+1}^{-1}}{d \mu}\left(T_{i} x\right)=\frac{1}{J_{\mu T_{i+1}}(x)}=\frac{d \mu T_{i+1}^{-1}}{d \mu}(T V x)=\frac{1}{J_{\mu T}(V x)} .
$$

Therefore

$$
\frac{d \mu V}{d \mu}(x)=\frac{J_{\mu T}(x)}{J_{\mu T}(V x)}
$$

Similarly, for $x \in A_{n}$,

$$
J_{\mu V}(x)=\frac{d \mu V}{d \mu}(x)=\frac{d \mu T_{1}^{-1}}{d \mu}\left(T_{n} x\right) \frac{d \mu T_{n}}{d \mu}(x)=\frac{J_{\mu T}(x)}{J_{\mu T}(V x)} .
$$

It is easy to check that $T \circ V=T$ a.e.

We can obtain a similar result for any $k \in \mathbb{N}$, by noting that the $V$ we define in the above theorem was only in the full group of the subrelation $S_{1}=$ $\{(x, y) \in X \times X: T x=T y\}$. The same proof as above gives us the following corollary. 
Corollary 3.3. Suppose $T$ is an $n$-to-1 nonsingular endomorphism of $(X, \mathscr{B}, \mu)$ and $k \in \mathbb{N}$. Then there exists $V \in[S], V \neq \mathrm{Id}$, such that $d \mu V / d \mu$ is a $V$-coboundary with transfer function $J_{\mu T^{k}}$ and $J_{\mu T^{k}}$ is the Jacobian for $T^{k}$. Furthermore $V$ is periodic of period $n^{k}$, and $T^{k} \circ V=T^{k}$ a.e.

Corollary 3.4. If $T$ is bounded-to-1, there exists $V \in[S], V \neq I d$, such that $d \mu V / d \mu$ is a coboundary for $V$ with transfer function $J_{\mu T}$, and $T \circ V=T$ a.e.

Proof. The construction of $V$ is only slightly more complicated than in the proof of 3.2. Suppose that $\zeta$ has $m$ atoms. We write $A_{1}$ as a disjoint union, $A_{1}=B_{1} \cup A_{1}^{2} \cup \cdots \cup A_{1}^{m}$, where $A_{1}^{i}=\left\{x \in A_{1}: \exists y \in A_{i}\right.$, s.t. $T x=T y$, but no $y$ exists for and $j<i\}$. The set $B_{1}=\left\{x: T x=T y \Rightarrow y \notin A_{i}\right.$ for any $\left.i>1\right\}$. We define $\left.V\right|_{B}=\mathrm{Id}$, and $\left.V\right|_{A_{i}}=T_{i}^{-1} \circ T$. We continue the algorithm, writing $A_{i}=B_{i} \cup A_{i}^{i+1} \cup \cdots \cup A_{i}^{m}$ for $i<m$, and map $A_{m}$ and all $B_{i}$ 's into $A_{1}$.

Remark 3.5. 1. Changing to a measure $\nu \sim \mu$ with $h=d \mu / d \nu$ gives $d \nu V / d \nu$ a coboundary with transfer function $J_{\mu T} h$. The choice of $V$ is not unique in the above results.

2. For $T$ bounded-to-1, we write $S=S^{1} \cup S^{2} \cup \cdots \cup S^{n} \ldots$ where $(x, y) \in$ $S^{i}$ if $T^{i} x=T^{i} y$ and $T^{j} x \neq T^{j} y$ for $j<i$. For each fixed $i$, Theorem 3.2 and Corollaries 3.3 and 3.4 give us a canonical method for defining an element of $V_{i} \in[S], V_{i} \in S^{i}$, which admits an equivalent invariant measure. Then $X /\left[S^{i}\right]=X / V_{i}$-orbits, and letting $\pi_{i}: X \rightarrow X /\left[S^{j}\right]=X_{i}$, we see that $T^{i} \circ \pi_{i}=T^{j}$ a.e. for all $i \in \mathbb{N}$. The factor algebra of measurable sets in $X_{i}$ is given by $\mathscr{B}_{i}=\left\{A \in \mathscr{B}: A=T^{-i} C\right.$ for some $\left.C \in \mathscr{B}\right\}$; that is, $\mathscr{B}_{i} \simeq T^{-i} \mathscr{B}$, so $\left(X_{i}, \mathscr{B}_{i}, \mu_{i}\right) \simeq\left(X, T^{-i} \mathscr{B}, \mu_{i}\right)$, where $\mu_{i}$ is the restriction of $\mu$ to $T^{-i} \mathscr{B}$. Clearly $\pi_{i}$ is nonsingular with respect to $\mu$ and $\mu_{i}$; i.e., it is a factor map for $T$. The endomorphism $T$ is exact if and only if only the trivial sets $\varnothing$ and $X$ are in $X_{i}$ for all $i$. The maps $T^{i}: X_{i} \rightarrow X$ are invertible.

3. More generally, let $V$ denote any partial isomorphism of $S$. Then $V \in$ [[S]], so if $V: A \rightarrow B$, then for every $x \in A, V x \in T^{-k(x)}\left(T^{k(x)} x\right)$. Then $d \mu V(x) / d \mu=J_{\mu T^{k(x)}}(x) / J_{\mu T^{k(x)}}(V x)$ by the above arguments. This tells us that the ratio set of $S$ can be computed by using ratios of the value of the Jacobians of the powers of $T$. If the Jacobian of $T^{k}$ is $T^{-k} \mathscr{B}$-measurable for all $k \in \mathbb{N}$, then the relation $S_{T}$ is type II, since $J_{\mu T^{k}}$ is completely determined on one atom of $\zeta_{k}$, and $(x, y) \in S \Rightarrow J_{\mu T^{k}}(x)=J_{\mu T^{k}}(y)$ for some $k$. Under the assumption that $\mu(X)=1, J_{\mu T^{k}}$ is $T^{-k} \mathscr{B}$-measurable for all $k$ implies that the relation $S_{T}$ is type $\mathrm{II}_{1}$.

We have just outlined the proof of the following corollary.

Corollary 3.6. If $T$ is an ergodic nonsingular endomorphism which is countableto-1, and the Jacobian $J_{\mu T}$ is constant a.e. on $X$, then the relation $S_{T}$ is of type II. If $\mu(X)<\infty$, then $S$ is of type $\mathrm{II}_{1}$; otherwise $S$ is of type $\mathrm{II}_{\infty}$.

Proof. By the above arguments, for any $V \in[[S]]$, we have

$$
\frac{d \mu V}{d \mu}(x)=\frac{J_{\mu T^{n(x)}}(x)}{J_{\mu T^{n(x)}}(V x)}=\frac{K^{n(x)}}{K^{n(x)}}=1, \quad \text { where } J_{\mu T}(x)=K \text { a.e. }
$$

That the converse to Corollary 3.6 is false can be seen in the following example. 
Example 3.7. We give an ergodic endomorphism $T$ whose Jacobian is nonconstant and satisfies, $J_{\mu T^{k}}$ is $T^{-k} \mathscr{B}$-measurable for each $k \geq 1$. It is a similar construction to Example 1.9; it is also a $C^{\infty}$ map on a torus. The space on which $T$ acts is $T^{2} \simeq \mathbb{R}^{2} / \mathbb{Z}^{2}$, endowed with the $\sigma$-algebra of Borel sets and Lebesgue measure. We define two maps of the circle, $g(x)=2 x(\bmod 1)$ and let $f$ be any orientation-preserving diffeomorphism of the circle which is Lebesgue ergodic. Then on $T^{2}$ we let $T(x, y)=(g(x), f(y))=(2 x(\bmod 1), f(y))$. Since $g$ is exact and $f$ is ergodic, $T$ is ergodic by [1].

We compute the Jacobian to be $J_{\mu T}(x, y)=2 D f\left(f^{-1} y\right)=2 / D f^{-1}(y)$, which is clearly $T^{-1} \mathscr{B}$-measurable. Furthermore, for any $k \geq 1$,

$$
J_{\mu T^{k}}(x, y)=2^{k} D f^{k}\left(f^{-k} y\right)=2^{k} / D f^{-k}(y),
$$

which is $T^{-k} \mathscr{B}$-measurable. This example appears in a paper by the author and Silva [12]. Obviously, the Jacobian will not be constant unless $f$ is chosen to be irrational rotation as in Example 1.9.

Corollary 3.8. If $T$ is an ergodic nonsingular endomorphism which is boundedto-1, and the Jacobian $J_{\mu T}$ is constant a.e. on $X$, then the relation $R_{T}$ is of type $\mathrm{III}_{K}$ and $K=J_{\mu T}(x)$ a.e.

Proof. By the above arguments, for any $V \in[[R]]$, we have

$$
\begin{aligned}
& \frac{d \mu V}{d \mu}(x)=\frac{J_{T^{m(x)}}(x)}{J^{T^{n(x)}}(V x)}=\frac{K^{m(x)}}{K^{n(x)}}=K^{m(x)-n(x)} \quad \text { a.e. } \square \\
& \text { 4. THE RADON-NIKODYM DERIVATIVE AND EQUIVALENT } \\
& \text { INVARIANT MEASURES FOR } T
\end{aligned}
$$

In this section we study some connections between the Jacobian and the Radon-Nikodym derivative of the endomorphism $T$ itself, not the associated relations. Throughout this section we will assume that $T$ is a finite-to-1 (but not necessarily bounded-to-1) nonsingular ergodic endomorphism of a Lebesgue space with $\mu$ a $\sigma$-finite measure. Since $\mu$ is $\sigma$-finite on $\mathscr{B}, \mu$ is also $\sigma$-finite on $T^{-1} \mathscr{B}$, and on $T^{-n} \mathscr{B}$ for all $n \geq 1$. There are several equivalent ways to define the Radon-Nikodym derivative of $T$ which we will denote by $\omega_{\mu}$; they have been described in some detail by Silva [23]. We will define $\omega_{\mu}$ to be the unique $T^{-1} \mathscr{B}$-measurable function satisfying

$$
\int_{X} f \circ T \cdot \omega_{\mu} d \mu=\int_{X} f d \mu \quad \text { for all } f \in L^{1}(X, \mu) .
$$

We define the cocycle generated by $\omega_{\mu}$ by

$$
\omega_{\mu}(k, x)=\omega_{\mu}(x) \omega_{\mu}(T x) \cdots \omega_{\mu}\left(T^{k-1} x\right) \quad \text { for } k \in \mathbb{N}, x \in X .
$$

Alternatively, if one writes $\theta_{\mu}(x)=d \mu T^{-1}(x) / d \mu$, then obviously $\theta_{\mu} \circ T$ is $T^{-1} \mathscr{B}$-measurable, and by uniqueness of Radon-Nikodym derivatives, $\omega_{\mu}(x)=$ $1 / \theta_{\mu}(T x)$ a.e. Furthermore, one can check by writing down the integral equations that

and

$$
\theta_{\mu}(x)=\sum_{y \in T^{-1} x} \frac{1}{J_{\mu T}(y)}
$$

$$
\omega_{\mu}(x)=\frac{1}{\sum_{y \in T^{-1}(T x)} 1 / J_{\mu T}(y)} .
$$


Definition 4.1. (1) By the Jacobian cocycle for $T$ (w.r.t. $\mu$ ) we mean the cocycle $J_{\mu}: \mathbb{N} \times X \rightarrow \mathbb{R}^{+}$generated by the Jacobian function for $T$ defined in $\S 3$; i.e., $J_{\mu}(1, x)=J_{\mu T}(x)$ for a.e. $x \in X$, and $J_{\mu}(k, x)=\prod_{i=0}^{k-1} J_{\mu T}\left(T^{i} x\right)=J_{\mu T^{k}}(x)$ a.e. for all $k \in \mathbb{N}$.

(2) The index function for a nonsingular endomorphism $T$ which is finiteto-1 is the $T^{-1} \mathscr{B}$-measurable function $n: X \rightarrow \mathbb{N}$ which for each $x$ gives the number of preimages of $T x$. The map $T$ is $n(x)$-to-1 at $x$, and we call the set of preimage points of $T x,\left\{x=x_{1}, x_{2}, \ldots, x_{n(x)}\right\}$, symmetric points for $x$, or just symmetric points. The function $n$ generates an $\mathbb{N}$ cocycle for $T$, and $n(k, x)=n_{T^{k}}(x)$ for all $k \in \mathbb{N}$. If $T$ is an $n$-to-1 endomorphism, then $n(k, x)=n^{k}$ a.e. This function was defined by Walters [25].

Definition 4.2. The operators $P_{\mu}^{k}$ and $E_{\mu}^{k}$. We define the following operators which are generalizations of the Perron-Frobenius operator to the nonsingular case.

We fix any $k \in \mathbb{N}$. For each measurable function $f$, we define the function

$$
P_{\mu}^{k} f(x)=\sum_{y \in T^{-k}\left(T^{k} x\right)} \frac{f(y)}{J_{\mu T^{k}}(y)} .
$$

(1) The operator $P_{\mu}^{k}$ takes measurable functions on $(X, \mathscr{B}, \mu)$ to measurable functions, and $P_{\mu}^{k} f$ is $T^{-k} \mathscr{B}$-measurable for any $\mathscr{B}$-measurable $f$.

(2) $P_{\mu}^{k}$ is linear in $f$; if $f \geq 0$, then $P_{\mu}^{k} f \geq 0$.

(3) We have that $\omega_{\mu}=1 / P_{\mu}^{1} 1$, and for every $k \in \mathbb{N}, P_{\mu}^{k}(1)(x)=\left(\omega_{\mu T^{k}}\right)^{-1}(x)$ $\mu$ a.e.

We now define for each measurable $f$, and each $k \in \mathbb{N}$,

$$
E_{\mu}^{k}(f)=P_{\mu}^{k} f / P_{\mu}^{k} 1=P_{\mu}^{k} f \cdot \omega_{\mu} .
$$

The operator is a generalization of the conditional expectation operator onto the sub- $\sigma$-algebra $T^{-k} \mathscr{B}$, and

(4) When $f \in L^{1}(X, \mathscr{B}, \mu)$, we have $E_{\mu}^{k}(f)=E_{\mu}\left(f \mid T^{-k} \mathscr{B}\right)$.

(5) If $f$ is $T^{-k} \mathscr{B}$-measurable, then $E_{\mu}^{k}(f)=f$ and $P_{\mu}^{k}(f)=f / \omega_{\mu} T^{k}$.

(6) If $\mu$ is $T$-invariant, then for every measurable $f, P_{\mu}^{k}(f)=E_{\mu}^{k}(f)$.

(7) The operators $P_{\mu}^{k}=E_{\mu}^{k}$ are both well defined when $T$ is finite-to-1 and $\mu$ is infinite but $\sigma$-finite.

(8) For countable-to-1 endomorphisms, the operators are defined on $L^{1}(X, \mathscr{B}, \mu)$ if $\mu$ is a finite measure. This is discussed more in $\S 6$.

Definition 4.3. For a measurable function $a: X \rightarrow \mathbb{R}$ we consider the induced cocycle for $T$ given by $a(k, x)=\prod_{i=1}^{k-1} a\left(T^{i} x\right)$. Given two $\mathbb{N}$-cocycles for $T$, $a$ and $b$, we say that $a$ is conditionally cohomologous to $b$ if there exists a $\mathscr{B}$-measurable function $h$, called the transfer function such that

$$
\frac{a}{b}(x)=\frac{E_{\mu}^{1}(h \circ T)}{E_{\mu}^{1}(h)}(x)=\frac{h \circ T}{E_{\mu}^{1}(h)}(x) \quad \text { for } \mu \text { a.e. } x \in X .
$$

We note that this does not define an equivalence relation on cocycles, since it does not follow that there is some measurable function $g$ such that

$$
\frac{b}{a}(x)=\frac{g \circ T}{E_{\mu}^{1}(g)}(x) \quad \text { for } \mu \text { a.e. } x \in X
$$


instead by setting $g=1 / h$ we have that

$$
\frac{b}{a}(x)=g \circ T \cdot E_{\mu}^{1}\left(\frac{1}{g}\right)(x)=\frac{g \circ T}{E_{g d \mu}^{1}(g)}
$$

(this follows from Lemma 4.5).

From $(*)$ and $\left(*^{\prime}\right)$ we obtain the identity

$$
\frac{1}{E_{\mu}^{1}(h)}(x)=E_{\nu}^{1}\left(\frac{1}{h}\right)(x) \quad \text { with } \nu=\frac{1}{h} d \mu .
$$

A conditional coboundary (for $T$ and $\mu$ ) is a cocycle which is conditionally cohomologous to 1 ; that is, $a(x)=\left[(h \circ T) / E_{\mu}^{1}(h)\right](x)$ a.e. The conditionally cohomologous cocycles $a$ and $b$ are cohomologous if $h$ is $T^{-1} \mathscr{B}$-measurable. The following statements hold for conditional cohomology.

Remarks 4.4. (1) If $a$ is conditionally cohomologous to $b$ with transfer function $h$, then

$$
\frac{a}{b}(x)=\frac{h \circ T}{h}(x) \cdot \frac{h}{E_{\mu}^{1}(h)}(x) \quad \text { for } \mu \text { a.e. } x \in X .
$$

If we define the cocycle $h_{*}=h / E_{\mu}^{1}(h)$, then for all $k \in \mathbb{N}$,

$$
\frac{a}{b}(k, x)=\frac{h \circ T^{k}}{h}(x) \cdot h_{*}(k, x) \quad \text { for } \mu \text { a.e. } x \in X .
$$

(2) If $a$ and $b$ are conditionally cohomologous, then $a$ is $T^{-1} \mathscr{B}$-measurable if and only if $b$ is.

(3) A conditional coboundary is $T^{-1} \mathscr{B}$-measurable, but not necessarily recurrent. (See Example 4.13.1.) Therefore, not all conditional coboundaries are coboundaries.

(4) A conditional coboundary with transfer function $h$ is recurrent if and only if $h_{*}$ is. The cocycle $h_{*}$ can be characterized as a Radon-Nikodym derivative when $h=d \nu / d \mu$ for two finite equivalent measures. In particular, we denote by $\mu_{1}$ the factor measure on $\left(X_{1}, \mathscr{B}_{1}\right) \simeq\left(X, T^{-1} \mathscr{B}\right)$, as in Remark 3.5.2, then for a.e. $x \in X_{1}$ we have a measure defined on the atom of $x$ in $X$, say $\mu_{x}^{\mathscr{B}}$ (cf. [3]). A computation shows that for $\mu=h d \mu$ we have

$$
\frac{h}{E_{\mu}^{1}(h)}(x)=\frac{d \nu_{x}^{\mathscr{B}_{1}}}{d \mu_{x}^{\mathscr{B}_{1}}}(x)=\frac{d \nu}{d \mu} / E_{\mu}\left(\frac{d \nu}{d \mu} \mid \mathscr{B}_{1}\right)(x) .
$$

The above remarks suggest that the only impediments to the usual cohomology theory for invertible maps under change to equivalent measure are changes of distribution within individual atoms of $T^{-k} \mathscr{B}$.

Lemma 4.5. Assume that $T$ is finite-to-1 and $\nu$ and $\mu$ are both nonsingular $\sigma$-finite measures for $T$. The measure $\nu$ is equivalent to $\mu$ if and only if $\omega_{\nu}$ is conditionally cohomologous to $\omega_{\mu}$ (via $E_{\mu}^{1}$ ) if and only if $\omega_{\mu}$ is conditionally cohomologous to $\omega_{\nu}$ (via $\left.E_{\nu}^{1}\right)$.

Proof. Using the fact that $J_{\nu T}=[(h \circ T) / h] \cdot J_{\mu T}$, an easy computation shows that $\omega_{\nu} / \omega_{\mu}=(h \circ T) / E_{\mu}^{1}(h)$ with $h=d \nu / d \mu$. Similarly, $\omega_{\mu} / \omega_{\nu}=$ $\left(h^{-1} \circ T\right) / E_{\nu}^{1}\left(h^{-1}\right)$. 
It is well known and easily checked (cf. [22]) that $T$ admits a $\sigma$-finite invariant measure $\nu \sim \mu$ if and only if there exists a $\mathscr{B}$-measurable function $h$ satisfying $h(x)=\sum_{y \in T^{-1}(x)} h(y) / J_{\mu T}(y)$; this holds if and only if $h \circ T=P_{\mu}^{1} h$ a.e. The invariant measure $\nu$ is finite if and only if $h \in L^{1}(X, \mathscr{B}, \mu)$. This leads to the following theorem.

Theorem 4.6. If $T$ is a nonsingular finite-to-1 endomorphism of $(X, \mathscr{B}, \mu)$, then $\omega_{\mu}(x)=E_{\mu}^{1}\left(J_{\mu T}\right)(x) / n(x)$ and

(1) $\omega_{\mu}^{-1}=\theta_{\mu} \circ T$ is a conditional coboundary if and only if $T$ admits a $\sigma$-finite invariant measure equivalent to $\mu$.

(2) The index function $n(\cdot)$ is conditionally cohomologous to $E_{\mu}^{1}\left(J_{\mu T}\right)$ iff $T$ admits a $\sigma$-finite invariant measure equivalent to $\mu$.

Proof. We first see that

$$
E_{\mu}^{1}\left(J_{\mu T}\right)(x)=P_{\mu}^{1}\left(J_{\mu T}\right) \cdot \omega_{\mu}(x)=\sum_{y \in T^{-1}(T x)} 1 \cdot \omega_{n}(x)=n(x) \omega_{\mu}(x) .
$$

(1) $\Leftrightarrow)$ Assume that $\omega_{\mu}(x)=\left[E_{\mu}^{1}(h) /(h \circ T)\right](x)$. Then $h \circ T \cdot \omega_{\mu}=P_{\mu}^{1} h \cdot \omega$, so $h \circ T=P_{\mu}^{1} h$ a.e. and $T$ preserves a measure $\sim \mu$.

$(\Leftrightarrow)$ If $h \circ T=P_{\mu}^{1} h$ a.e., then $h \circ T \cdot \omega=P_{\mu}^{1} h \cdot \omega$ a.e. from which it follows immediately that $\omega_{\mu}(x)=\left[E_{\mu}^{1}(h) /(h \circ T)\right](x)$.

(2) This follows immediately from the first two statements.

Corollary 4.7. $T$ preserves $\mu$ if and only if $E_{\mu}^{1}\left(J_{\mu T}\right)(x)=n(x)$ a.e.

Proof. Apply the proof of Theorem 4.6(1), using $h=1$.

Corollary 4.8. If $T$ is $n(x)$-to-1 and the Jacobian of $T$ is $T^{-1} \mathscr{B}$-measurable then $T$ admits a $\sigma$-finite invariant measure $\sim \mu$ iff $n(\cdot)$ is conditionally cohomologous to $J_{\mu T}$.

Corollary 4.9. Let $f$ be a piecewise $C^{1}$ map of a bounded interval I onto itself which is symmetric in $x$ (so that the Jacobian is $T^{-1} \mathscr{B}$-measurable). Then $f$ admits an invariant measure equivalent to Lebesgue measure if and only if $\left|f^{\prime}\right|$ is conditionally cohomologous to $n(x)$.

We now explore some further connections between the two cocycles $J_{\mu T}$ and $\omega_{\mu}$. By $r_{\mu}(T)$ we mean the usual Krieger ratio set (cf. [9] and [12]) for noninvertible maps.

Proposition 4.10. Let $T$ be a conservative nonsingular finite-to-1 endomorphism of $(X, \mathscr{B}, \mu)$ admitting a $\sigma$-finite invariant measure $\nu \sim \mu$. Let $h=d \nu / d \mu$. The following statements are equivalent.

(1) $\omega_{\nu}$ is cohomologous to $\omega_{\mu}$.

(2) $\omega_{\mu}$ is a coboundary

(3) $h$ is $T^{-1} \mathscr{B}$-measurable.

(4) $E_{\mu}^{1}\left(J_{\mu T} \mid T^{-1} \mathscr{B}\right)$ is cohomologous to $n(\cdot)$ with transfer function $h$.

(5) $E_{\mu}^{1}\left(J_{\mu T} / n(\cdot) \mid T^{-1} \mathscr{B}\right)$ is coboundary with transfer function $h$.

(6) $\omega_{\mu}$ is a recurrent cocycle for $T$.

(7) $r_{\mu}(T)=\{1\}$.

(8) $h_{*}$ is a recurrent cocycle.

Proof. (1) $\Leftrightarrow(2)$ is trivial and (2) $\Leftrightarrow(3)$ is from [11]. 
(3) $\Rightarrow$ (4) If $T$ admits a $\sigma$-finite invariant measure $\nu \sim \mu$, then

$$
h \circ T(x)=\sum_{y \in T^{-1}(T x)} \frac{h(y)}{J_{\mu T}(y)}=h(x) \sum_{y \in T^{-1}(T x)} \frac{1}{J_{\mu T}(y)}
$$

by our hypothesis on $h$. Then it follows immediately that

$$
(h \circ T) / h=n(\cdot)\left[E_{\mu}^{1}\left(J_{\mu T}\right)\right]^{-1} .
$$

$(4) \Leftrightarrow(3)$ is clear.

To show that $(4) \Leftrightarrow(5)$, we note that $n(\cdot)\left[E_{\mu}^{1}\left(J_{\mu T}\right)\right]^{-1}(x)=1(x) / \omega_{\mu}$. Since $\left.E_{\mu}^{1} J_{\mu T} / n(\cdot)\right)=\omega_{\mu}$ and coboundaries are recurrent, (6) follows from (5).

$(1) \Rightarrow(6)$ is proved in [11], (6) $\Rightarrow(1)$ is proved in [24], and $(6) \Rightarrow(7)$ is done in [12]. (7) $\Rightarrow(6)$ trivially, $(4) \Rightarrow(8)$ trivially, and $(8) \Rightarrow(6)$ by Lemma 4.5 .

From Remarks 4.4 we obtain the following.

Proposition 4.11. For $T$ a finite-to-1 endomorphism of $(X, \mathscr{B}, \mu), T$ admits a $\sigma$-finite measure $\nu=h d \mu$ :

(a) if and only if for every $k \in \mathbb{N}, E_{\mu}^{k}\left(\omega_{\mu}(k, x)\right)^{-1}$ is a conditional coboundary for $T^{k}$;

(b) if and only if

$E_{\mu}^{k}\left(\frac{h \circ T^{k}}{h} \cdot h_{*}(k, \cdot)\right)(x)=h \circ T^{k} \cdot E_{\mu}^{k}\left(\frac{h_{*}(k, \cdot)}{h}\right)(x)=h \circ T^{k} \cdot \frac{1}{E_{\mu}^{k}(h)}(x) \quad$ a.e.

Proof. (a) follows from the easily checked property that $\omega_{\mu}(k, x)$ is Markovian for $T^{k}$ [24], and Theorem 4.6.

(b) follows since $T^{k}$ preserves $h d \mu$ for all $k \in \mathbb{N}$ if it preserves $h d \mu$ for $k=1$.

Remarks 4.12. 1. We note that in general $\theta_{\mu}$ is not $T^{-1} \mathscr{B}$-measurable. A computation shows that

$$
E_{\mu}^{1}\left(\theta_{\mu}(x)=\frac{\omega_{\mu}}{\omega_{\mu T^{2}} \circ T^{-1}}(x) \quad \text { and } \quad E_{\mu}^{1}\left(\theta_{\mu} \circ T\right)(x)=\left[\omega_{\mu}(x)^{-1}\right] .\right.
$$

2. Under the assumption that $J_{\mu T^{k}}$ is $T^{-k} \mathscr{B}$-measurable for all $k \in \mathbb{N}$, we obtain chain rules for the cocycles generated by both $\omega_{\mu}$ and $\theta_{\mu}$; i.e., for $\mu$ a.e. $x$ and all $k \in \mathbb{N}$,

$$
\omega_{\mu}(k, x)=\omega_{\mu T^{k}}(x) \quad \text { and } \quad \theta_{\mu}(k, x)=\theta_{\mu T^{k}}(x) .
$$

This can occur without the Jacobian being constant as can be seen in Example 2.5 .

We conclude this section with some examples which illustrate the results of this section, including conditional coboundaries that are not coboundaries.

\section{Examples 4.13.}

1. The modified Boole transformation. By $(\mathbb{R}, \mathscr{B}, m)$ we denote the space of real numbers with the usual Borel structure and Lebesgue measure. We consider the map $T x=\frac{1}{2}(x-1 / x)$ which is defined and 2-to-1 (w.r.t. $m$ ) on $\mathbb{R}$. Straightforward computations show that $T x=T(-1 / x)$, and $\omega_{m}(x)=$ $\omega_{m}(-1 / x)=\frac{1}{2}$ for a.e. $x$. Clearly $m$ is not a recurrent measure, and $\omega_{m}$ is not a coboundary. However, it is well known that $T$ preserves the probability 
measure $2 / \pi\left(1+x^{2}\right) d x$ (cf. [7]). One can verify Theorem 4.6 directly by computing that if $h(x)=2 / \pi\left(1+x^{2}\right)$, then

$$
\left[\omega_{m}(x)\right]^{-1}=2=\frac{h \circ T}{E_{m}^{1}(h)}(x)=\frac{8 x^{2}}{\pi\left(x^{2}+1\right)^{2}} \frac{\pi\left(x^{2}+1\right)^{2}}{4 x^{2}} \quad \text { a.e. }
$$

That is, $\left[\omega_{m}\right]^{-1}$ is a conditional coboundary.

2. A modified transformation of Renyi. On the Lebesgue space $([0,1], \mathscr{B}, m)$ we define the transformation $T x=x /(1-x)$ for $x \in\left[0, \frac{1}{2}\right)$ and $T x=2 x-1$ for $x \in\left[\frac{1}{2}, 1\right)$. This is a 2-to-1 map which preserves the $\sigma$-finite infinite measure $\nu=1 / x d x$ (cf. [22]). For each $x \in\left[0, \frac{1}{2}\right.$ ), there corresponds the symmetric point $y=1 / 2(1-x) \in\left[\frac{1}{2}, 1\right)$ such that $T x=T y$. Similarly, each $y \in\left[\frac{1}{2}, 1\right)$ has a unique symmetric point $x=(2 y-1) / 2 y \in\left[0, \frac{1}{2}\right)$. We compute for $x \in\left[0, \frac{1}{2}\right), \omega_{m}(x)=2 /\left(2 x^{2}-4 x+3\right)$. We check that this is a conditional coboundary with transfer function $h(x)=1 / x$. For $x \in\left[0, \frac{1}{2}\right)$,

$$
h \circ T(x)=\frac{1-x}{x}, \quad \text { and } \quad P_{m}^{1}(h)(x)=\frac{1}{x}(1-x)^{2}+2(1-x) \frac{1}{2}=\frac{1-x}{x} .
$$

By this and 4.2(3) it follows immediately that $\omega_{m}^{-1}$ is a conditional coboundary with transfer function $h$, but $\omega_{m}$ is not a coboundary. From Proposition 4.10 we can also conclude that Lebesgue measure is not recurrent for $T$.

\section{RECURRENCE OF COCYCLES AND MEASURES FOR ENDOMORPHISMS}

We assume in this section that $T$ is conservative; i.e., for every set $A \in$ $\mathscr{B}, \mu(A)>0$, there exists $n \in \mathbb{N}$ such that $\mu\left(A \cap T^{-n} A\right)>0$. We also assume, by passing to a cohomologous measure if necessary that $\mu(X)=1$. This section consists of material which extends some work of Silva and Thieullen on Markovians [24].

Definition 5.1. Any positive measurable function $\omega$ satisfying

$$
\int_{X} f(T x) \cdot \omega(x) d \mu(x)=\int_{X} f(x) d \mu(x)
$$

for every $f \in L^{1}(X, \mathscr{B}, \mu)$ is said to be Markovian for $T$ and $\mu$.

Remarks. 1. It was shown in [24] that $\omega_{\mu T}$ is the unique $T^{-1} \mathscr{B}$-measurable function which is Markovian for $T$ and $\mu$.

2. If $\omega$ is a $\mathscr{B}$-measurable function which is Markovian for $T$ and $\mu$ then $\omega \in L^{1}(X, \mathscr{B}, \mu)$ and $E_{\mu}\left(\omega \mid T^{-1} \mathscr{B}\right)=\omega_{\mu}$. (Here $E_{\mu}\left(h \mid T^{-1} \mathscr{B}\right)$ denotes the usual conditional expectation of $h \in L^{1}(X, \mathscr{B}, \mu)$ with respect to the sub- $\sigma$ algebra $T^{-1} \mathscr{B}$.)

3. Similarly, $\omega_{\mu T^{k}}(x)=\left(d \mu / d \mu T^{-k}\right)\left(T^{k} x\right)$ is the unique $T^{-k} \mathscr{B}$-measurable function which is Markovian for $T^{k}$ and $\mu$. It is easily seen that $\omega_{\mu}(k, x) \equiv$ $\omega_{\mu T}(x) \omega_{\mu T}(T x) \cdots \omega_{\mu T}\left(T^{k-1} x\right)$ is a $\mathscr{B}$-measurable function which is Markovian for $T^{k}$ and $\mu$ so that $E_{\mu}\left(\omega_{\mu}(k, \cdot) \mid T^{-k} \mathscr{B}\right)=\omega_{\mu T^{k}}$.

Definition 5.2. Any Markovian $\omega$ (for $T$ and $\mu$ ) defines a cocycle $\omega: \mathbb{N} \times X \rightarrow$ $X$ as follows: for every $i \in \mathbb{N}, x \in X, \omega(i, x)=\prod_{k=0}^{i-1} \omega\left(T^{k} x\right)$. The cocycle 
$\omega$ is recurrent if for every $A \in \mathscr{B}, \mu(A)>0$, and every $\varepsilon>0$, there exists an $n \in \mathbb{N}$ such that $\mu\left(A \cap T^{-n} A \cap\{x:|\omega(n, x)-1|<\varepsilon\}\right)>0$. A Markovian $\omega$ is recurrent if the cocycle it generates under the $\mathbb{N}$ action of $T$ is recurrent. Equivalently, $\omega$ is recurrent if for $\mu$ a.e. $x \in X, \sum_{k=0}^{\infty} \omega(i, x)=\infty$ [23].

In the next proposition we characterize all Markovians for $T$ and $\mu$.

Proposition 5.3. The function $\omega$ is Markovian for $T$ and $\mu$ if and only if there exists a measurable function $h$ satisfying (i) $\omega(x)=h(x) \omega_{\mu}(x)$, (ii) $P_{\mu}^{1}(h)=$ $1 / \omega_{\mu}$, and (iii) $E_{\mu}^{1}(h)=1$. Furthermore, given any positive measurable function $g$ on $X$, the function $h=g / E_{\mu}^{1}(g)$ satisfies (ii) and (iii) and $\omega(x)=g / P_{\mu}^{1}(g)$ is Markovian.

Proof. $(\Rightarrow)$ Suppose $\omega$ is Markovian. Then

$$
E_{\mu}\left(\omega \mid T^{-1} \mathscr{B}\right)=E_{\mu}^{1}(\omega)=P_{\mu}^{1}(\omega) \omega_{\mu}=\omega_{\mu},
$$

so $P_{\mu}^{1}(\omega)=1$ and $h=\omega / \omega_{\mu}$ satisfies $P_{\mu}^{1}(h)=\frac{1}{\omega_{\mu}} P_{\mu}^{1}(\omega)=1 / \omega_{\mu}$. Consequently (i)-(iii) hold.

$(\Leftarrow)$ Now suppose that $h$ is any function such that $P_{\mu}^{1}(h)(x)=\left[\omega_{\mu}(x)\right]^{-1}$. Obviously (iii) holds then, and we show that $h \omega_{\mu}$ satisfies $(*)$. Given any nonnegative measurable function $f$,

$$
\int_{X} f(x) d \mu(x)=\int_{X} f \circ T(x) \cdot \omega_{\mu}(x) d \mu(x)
$$

since $\omega_{\mu}$ is Markovian; since $f \circ T$ is $T^{-1} \mathscr{B}$-measurable, and $E_{\mu}^{1}\left(h \omega_{\mu}\right)=$ $E\left(h \omega_{\mu} \mid T^{-1} \mathscr{B}\right)=\omega_{\mu}$, we have

$$
\int_{X} f \circ T(x) \cdot \omega_{\mu}(x) d \mu(x)=\int_{X} f \circ T(x) \cdot h \omega_{\mu}(x) d \mu(x) .
$$

To prove the second statement, consider any positive measurable function $g$. The function $h=g / E_{\mu}^{1}(g)$ clearly satisfies (ii) and (iii), so $h \omega_{\mu}$ is Markovian. But

as claimed.

$$
h \omega_{\mu}=\frac{g}{P_{\mu}^{1}(g) \omega_{\mu}} \omega_{\mu}=\frac{g}{P_{\mu}^{1}(g)}
$$

Proposition 5.4. Markovians for $T$ and $\mu$ are in one-to-one correspondence with $\sigma$-finite measures equivalent to $\mu$. Recurrent Markovians for $T$ and $\mu$ are in one-to-one correspondence with equivalent recurrent measures.

Proof. Suppose that $\omega$ is any Markovian for $T$ and $\mu$; then $E_{\mu}^{1}(\omega)=\omega_{\mu}$. We consider the measure $\nu=\omega d \mu$, and we have from Lemma 4.5 that $\omega_{\nu}=$ $\left[(\omega \circ T) / E_{\mu}^{1}(\omega)\right] \omega_{\mu}=\omega \circ T$. The measure $\nu$ is a recurrent measure if and only if $\omega_{\nu}$ is recurrent if and only if $\omega \circ T$ is a recurrent cocycle. But $\omega$ is cohomologous to $\omega \circ T$ so $\omega$ is a recurrent Markovian if and only if $\omega d \mu$ is a recurrent measure.

We now suppose that $\nu \sim \mu$ is any $\sigma$-finite equivalent measure. Then we let $f(x)=(d \nu / d \mu)(x)$; we claim that the function $\omega(x) \equiv \omega_{\nu}(x)[(f \circ T) / f](x)$ is Markovian. This is because $\omega_{\nu}=\left[(f \circ T) / E_{\mu}^{1}(f)\right] \omega_{\mu}$, so $\omega(x)=\left[f / P_{\mu}^{1}(f)\right](x)$ which is Markovian by Proposition 5.3. 
Corollary 5.5. Suppose that $\mu$ is a $\sigma$-finite nonsingular measure for the conservative endomorphism $T$ of $(X, \mathscr{B})$. The measure $\nu \sim \mu$ is recurrent if and only if $f / P_{\mu}^{1}(f)$ is recurrent where $f=d \nu / d \mu$.

If $T$ admits an equivalent invariant measure, then obviously the invariant measure is a recurrent one. The existence of an equivalent recurrent measure for each endomorphism is still open. Silva and Thieullen [24] showed that if $\mu$ is an invariant measure for $T$ (finite or $\sigma$-finite), then any equivalent recurrent measure is cohomologous to $\mu$. In other words, say $\nu=h d \mu$; then we know that $\omega_{\nu} / \omega_{\mu}=(h \circ T) / E_{\mu}^{1}(h)$. The result of Silva and Thieullen implies that $E_{\mu}^{1}(h)=h$ if and only if $\nu$ is recurrent. Since the same statement holds for all powers of $T$, we have the following result.

Proposition 5.6. Suppose that $T$ is exact, preserves a $\sigma$-finite measure $\mu$ and that $\nu \sim \mu$ is a measure which is recurrent for $T^{k}$ for all $k \in \mathbb{N}$. Then $\nu=C \cdot \mu$ for some constant $C$; if $\mu$ and $\nu$ are probability measures, then $\nu=\mu$.

Proof. Writing $\nu=h d \mu$, the recurrence of $\nu$ for $T^{k}$ implies that $h$ is $T^{-k} \mathscr{B}$ measurable for all $k \in \mathbb{N}$. By exactness and the Martingale theorem, we have that $h$ is constant $\mu$ a.e. If $\mu$ and $\nu$ are probability measures, then $h=1$. If $\mu$ is infinite, we induce $T$ on a set of finite measure.

\section{COUNTABLE-TO-1 ENDOMORPHISMS AND EXAMPLES}

We will assume for now that $(X, \mathscr{B}, \mu)$ is a Lebesgue probability space and that $T$ is a nonsingular countable-to-1 endomorphism; we also assume that the index function $n(x)=\infty$ for $\mu$ a.e. $x$. As before, we define the Radon-Nikodym derivatives of $T$ to be the unique $T^{-1} \mathscr{B}$-measurable function satisfying $\int f \circ T \cdot \omega_{\mu} d \mu=\int f d \mu$ for $f \in L^{1}(X, \mathscr{B}, \mu)$. Clearly $\omega_{\mu} \in L^{1}(X, \mathscr{B}, \mu)$. For $k \in \mathbb{N}$, the operators $E_{\mu}^{k}$ and $P_{\mu}^{k}$ are well defined on $L^{1}(X, \mathscr{B}, \mu)$ as follows; we just define $E_{\mu}^{k}$ to be the usual conditional expectation operator onto $T^{-k} \mathscr{B}$ and, we define $P_{\mu}^{k}(f)(x)=E_{\mu}^{k}(f) / \omega_{\mu T^{k}}(x)$. When the measure is infinite or the function is not integrable, these operators can fail to give finite-valued functions. We consider a few examples below.

All examples considered are nonsingular and piecewise linear. The first example was shown to the author by Klaus Schmidt [21]. It is an example of an endomorphism $T$ with the following properties: (1) $T$ is a countable-to-1 endomorphism of the nonnegative reals; (2) the relation $R_{T}$ is ergodic $\mathrm{II}_{\infty}$, and $S_{T}$ is $\mathrm{II}_{\infty}$ as well though not ergodic; (3) $T$ preserves no $\sigma$-finite measure equivalent to Lebesgue measure; (4) $T$ is dissipative.

This example contrasts with the following easily proved lemma from ergodic theory.

Lemma 6.1. If $T$ is an invertible, nonsingular, ergodic transformation of a $\sigma$ finite Lebesgue space, then it is conservative.

Example 6.2 [21]. Let $X$ be the space of nonnegative real numbers endowed with the $\sigma$-algebra of Borel sets and set $m=$ Lebesgue measure. We represent any point in $X=\{x: x \geq 0\}$ by it decimal expansion; we then define $T$ by removing the leftmost nonzero digit, and replacing it by a zero. That is, if we write $x=a_{n} 10^{n}+\cdots+a_{0}+a_{-1} 10^{-1}+\cdots+a_{-m} 10^{-m}+\cdots$, with $a_{n} \neq 0$, then 
$T x=a_{n-1} 10^{n-1}+\cdots+a_{m} 10^{-m}+\cdots$. Equivalently, $T x=x-a_{n(x)} 10^{n(x)}$, where $n(x)=\max \left\{n \in \mathbb{Z}: a_{n} \neq 0\right.$ for $\left.x=\sum_{n=-\infty}^{+\infty} a_{n} 10^{n}\right\}$. We define $T(0)=0$.

We note that the set $E=[0,1)$ satisfies $E \subset T^{-1} E$ and the set $F=(1, \infty)$ satisfies $T^{-1} F \subset F$. Both containments are proper since $x=10 \in T^{-1} E$, but $x \notin E$ and $x=1.1 \in F$, but $x \notin F$. From this it follows that $T$ admits no $\sigma$-finite invariant measure $\nu \sim m$. If so, then $\nu\left(T^{-1} E\right)=\nu(E)$; hence $\nu\left(T^{-1} E \backslash E\right)=0$. But $E \neq T^{-1} E \Rightarrow m\left(T^{-1} E \backslash E\right)>0 \Leftrightarrow \nu\left(T^{-1} E \backslash E\right)>0$. This shows that there is no absolutely continuous invariant measure $\nu \ll \mu$.

We next show that $T$ is dissipative; we produce wandering sets of positive measure. To do this, we just consider any interval of the form $[n, n+1)$, for $n \in \mathbb{N}$. Then $T^{-1}[n, n+1) \subseteq[10 n, \infty)$. Suppose $x \in T^{-i} C \cap T^{-j} C, i \neq j$. Then $x \in T^{-i}[n, n+1) \Rightarrow x$ has exactly $i$ nonzero digits lying to the left of 1. $a_{-1} a_{-2} \ldots$. Similarly, $x$ must have exactly $j$ nonzero digits appearing to the left of $1 \ldots$ in its decimal expansion. This is obviously a contradiction unless $i=j$.

We now show the following.

Proposition 6.3. $T$ is ergodic with respect to Lebesgue measure.

Proof. Suppose that $T^{-1} A=A$ and $m(A)>0$. Then $T^{-1} A=A$ implies $T^{n} A=A$ for all $n \in \mathbb{Z}$. So $A$ must contain part of $[0,1)$ since $T^{n} x \rightarrow 0$ for all $x$. Also $A$ must contain most of some interval, i.e., by a Lebesgue density argument, there exists a rational number $a$, and $\varepsilon_{0}<1$, such that $m(A \cap[a, a+\varepsilon))>.99 \varepsilon$ for all $0<\varepsilon \leq \varepsilon_{0}$. Hence $m(A \cap[0, \varepsilon))>0$ since, $[a, a+\varepsilon)$ gets mapped to $[0, \varepsilon)$ under finitely many iterations of $T$. It can be easily checked that for any $\varepsilon>0, T^{-n}[0, \varepsilon)$ is all of $\mathbb{R}^{+}$. Since we found the original interval $[a, a+\varepsilon)$ to be mostly in $A$, letting $\varepsilon \rightarrow 0$, we see that $m\left(\mathbb{R}^{+} \backslash A\right)=0$.

We consider the relations $R_{T}$ and $S_{T}$ for this example. Since $T$ is ergodic and nonsingular, $R$ is also ergodic and nonsingular. We claim that $R$ and $S$ are both type $\mathrm{II}_{\infty}$; in particular Lebesgue measure is preserved. $R$ is ergodic because $T$ is; $S$ is not ergodic because we show that $T$ is not exact. We compute $\operatorname{Tail}(A)=\bigcup_{n=1}^{\infty} T^{-n}\left(T^{n} A\right)$ for sets $A$ of positive measure and show that we do not always get a tail of full measure. Letting $A=[0,1)$, for example, we see that while $\mu(\operatorname{Tail}(A))=\infty, \mu\left(\mathbb{R}^{+} \backslash \operatorname{Tail}(A)\right)=\infty$ as well.

An easy calculation shows that $J_{\mu}(n, x)=1$ for almost all $x$. The fact that the Jacobian is constant gives us that $S$ is type II by Corollary 3.6; the fact that the constant value is 1 tells us that $R$ is type II. Since Lebesgue measure is infinite we obtain $\mathrm{II}_{\infty}$ relations for both $R$ and $S$.

Example 6.4. Two strongly equivalent endomorphisms. We construct a countableto-1 map $T$ on the unit interval which preserves Lebesgue measure $m$ and for which $S$ is type $\mathrm{III}_{1 / 2}$. In this case, $\omega_{m}=1, h_{m}(T)=\infty$, and it can be shown that $T$ is strongly orbit equivalent to a 2-to-1 map of finite entropy. Let $X=[0,1), \mathscr{B}$ the $\sigma$-algebra of Borel sets, and $m=$ Lebesgue measure. On the interval $\left[\frac{1}{2}, 1\right)$, the graph of $T$ has slope 2 , on $\left[\frac{1}{4}, \frac{1}{2}\right)$ the graph has slope 4 , etc. On the interval $\left[1 / 2^{k}, 1 / 2^{k-1}\right)$, the graph has slope $2^{k}$, and for each piece of the graph, we draw the line segment passing through the points $\left(1 / 2^{k}, 0\right)$ and $\left(1 / 2^{k-1}, 1\right)$, leaving out the right-hand point of the line segment. 
This map can be described as follows: on the space $X=\prod_{i=0}^{\infty}\{0,1\}_{i}$, we put the usual Borel structure, the $\left(\frac{1}{2}, \frac{1}{2}\right)$ i.i.d. Bernoulli measure, and let $\sigma$ denote the one-sided shift. For $x \in X$, let $r(x)=\min \left\{j: x_{j}=1\right\}$; we then define $T x=\sigma^{r(x)} x$. We define $T(0)=0$.

We consider now the map $U$ on $I$ by $U(x)=3 x(\bmod 1)$ if $x \in\left[0, \frac{1}{3}\right)$ and $U(x)=\frac{3}{2} x(\bmod 1)$ if $x \in\left[\frac{1}{3}, 1\right)$, then we see that $R_{U}$ and $S_{U}$ are both of type $\mathrm{III}_{1 / 2}$, so $U$ and $T$ are strongly orbit equivalent. In both cases, $\omega=1$.

\section{SOME CONNECTIONS AND OPEN PROBLEMS}

We assume in this section that $\mu$ is a probability measure on $X$, and $T$ is a nonsingular, conservative, countable-to- 1 endomorphism of $X$. We consider any element $\phi \in\left[S_{T}\right]$. Then $\phi$ is invertible a.e., and for each $x \in X$ we have that $\phi(x)=T_{i(x)}^{-k(x)}\left(T^{k(x)} x\right)$, where by $T_{i(x)}$ we denote $\left.T\right|_{A_{i(x)}}$ with $A_{i(x)} \subset$ $\zeta_{k(x)}=\bigvee_{i=1}^{k(x)-1} T^{-i} \mathscr{P}$.

Definition 7.1. We define $\phi \in\left[S_{T}\right]$ to be of power $K$ (and write $\phi \in\left[S_{T}^{K}\right]$ or $\phi \sim K)$ if:

(i) $k(x)=K$ for a.e. $x$ in the above representation of $\phi$,

(ii) for all $k>K, T^{k} x \neq T^{k} \phi x$.

One constructs an automorphism $\phi$ of power $K$ by cutting one atom of the finite partition $\zeta_{K}$ into measurable subsets, cutting the rest of the atoms in the same way, and then permuting the subsets of the atoms among the other atoms.

We say $\phi$ is of power $\leq K$ (and write $\phi \leq\left[S_{T}^{K}\right]$ or $\phi \leq K$ ) if $k(x) \leq K$ for a.e. $x$ and $K$ is the minimum element of $\mathbb{N}$ with that property. If $\phi \leq K$, then $\phi$ can be expressed formally as an automorphism of power $K$ except that condition (ii) will fail to hold. We define $\phi$ to be of finite power if $\phi$ is of power $K$ for some $K>\infty$.

If $\phi \leq\left[S_{T}^{K}\right]$, for each measurable function $f$ we consider the operator $P_{\mu}^{K}$ defined in $\S 4$ :

$$
P_{\mu}^{K}(f)(x)=\sum_{y \in T^{-K}\left(T^{K} x\right)} \frac{f(y)}{J_{\mu T^{K}}(y)} .
$$

Proposition 7.2. For each $\phi \leq K$, the following hold:

(1)

$$
P_{\mu}^{K}\left(\frac{d \mu \phi}{d \mu}\right)(x)=P_{\mu}^{K}(1)(x)=\left[\omega_{\mu T^{k}}(x)\right]^{-1},
$$

(2) $(d \mu \phi / d \mu) \omega_{\mu T^{k}}$ is Markovian for $T^{k}$.

Proof. If $\phi \leq K$, then for a.e. $x, \phi(x)=T_{i(x)}^{-k(x)}\left(T^{k(x)} x\right)$, with $k(x) \leq K$. Because of the cancellation which occurs if $k(x)<K$ we have for $\mu$ a.e. $x$, $(d \mu \phi / d \mu)(x)=J_{\mu T^{K}}(x) / J_{\mu T^{\kappa}}(\phi x)$. By Corollary 3.4

$$
P_{\mu}^{K}\left(\frac{d \mu \phi}{d \mu}\right)(x)=\sum_{y \in T^{-K}\left(T^{K} x\right)} \frac{(d \mu \phi / d \mu)(y)}{J_{\mu T^{K}}(y)}=\sum_{y \in T^{-K}\left(T^{K} x\right)} \frac{1}{J_{\mu T^{K}}(\phi y)} .
$$

By assumption $\phi$ is a measurable automorphism, so by ignoring a set of measure zero on which $\phi$ fails to be invertible, we have that if $y, z \in T^{-K}\left(T^{K} x\right)$ and 
$y \neq z$, then $\phi y \neq \phi z$. Furthermore, by our representation of $\phi$, we know that $\phi y \in T^{-K}\left(T^{K} x\right)$ if $y$ is. By the surjectivity of $\phi$, every $y \in T^{k}\left(T^{K} x\right)$ is of the form $y=\phi z$ for some $z$, and since $T^{K} z=T^{K} y$, we have that $z \in T^{-K}\left(T^{K} x\right)$ as well. Therefore, we can write

$$
\sum_{y \in T^{-K}\left(T^{K} x\right)} \frac{1}{J_{\mu T^{K}}(\phi y)}=\sum_{y \in T^{-K}\left(T^{K} x\right)} \frac{1}{J_{\mu T^{K}}(y)}=\left[\omega_{\mu}^{K}(x)\right]^{-1} \text { as claimed. }
$$

We have the following proposition.

Proposition 7.3. If $T$ is an ergodic conservative finite-to-1 endomorphism on $(X, \mathscr{B}, \mu)$, then the following are equivalent:

1. $T$ preserves $\mu$.

2. For some $\phi \in\left[S_{T}^{1}\right], P_{\mu}^{1}(d \mu \phi / d \mu)(x)=1$ a.e.

3. For all $K \in \mathbb{N}$, for all $\phi \leq K, P_{\mu}^{K}(d \mu \phi / d \mu)(x)=1$ a.e.

Proof. This is clear from Proposition 7.2(1) and the fact that $T$ preserves $\mu$ iff $\omega_{\mu T}(x)=1$ a.e. iff $\omega_{\mu}^{K}(x)=1$ a.e. for all $K \geq 1$.

In what follows we will only consider $\phi \in\left[S_{T}\right]$ of finite power, so we can assume $\phi$ is conservative (because it is periodic). For conservative $\phi$, by [19] we have that $d \mu \phi / d \mu$ is a recurrent cocycle for (the integer action generated by) $\phi$. By Proposition 7.2 $E_{\mu}^{K}(d \mu \phi / d \mu)$ is also a recurrent cocycle for $\phi$ and for $T$ (since it is identically 1 ), even if $\mu$ is not a recurrent measure for $T$. The proposition below illustrates the effect of changing to an equivalent measure.

Proposition 7.4. Assume $T$ is a conservative ergodic finite-to-1 endomorphism of $(X, \mathscr{B}, \mu), \phi \in\left[S_{T}\right]$ is of power $K$, and $\nu=h d \mu$. Then

1. The cocycle $d \nu \phi / d \nu$ is cohomologous to $d \mu \phi / d \mu$ with transfer function $h$.

2. The function $P_{\mu}^{K}(d \mu \phi / d \mu)$ is conditionally cohomologous to $P_{\nu}^{K}(d \nu \phi / d \nu)$ as a $T^{K}$ cocycle with transfer function $h$.

3. The cocycle $P_{\nu}^{K}(d \nu \phi / d \nu)$ is cohomologous to $P_{\mu}^{K}(h) / h$ under $T^{K}$. Equivalently, $\omega_{\nu}^{K}$ is cohomologous to $h / P_{\mu}^{K}(h)$.

4. Recurrence of the measure $\nu$ for $T^{K}$ is equivalent to recurrence of the $T^{K}$ cocycle $h / P_{\mu}^{K}(h)$.

Proof. 1. This follows from Schmidt [19] since $\phi$ is an automorphism.

2.

$$
\begin{aligned}
P_{\mu}^{K}\left(\frac{d \nu \phi}{d \nu}\right) & =\left[\omega_{\nu}^{K}\right]^{-1}=\frac{E_{\mu}^{K}(h)}{h \circ T^{K}}\left[\omega_{\mu}^{K}\right]^{-1} \quad(\text { by Lemma 4.5) } \\
& =\frac{E_{\mu}^{K}(h)}{h \circ T^{K}} P_{\mu}^{K}\left(\frac{d \mu \phi}{d \mu}\right) .
\end{aligned}
$$

3. By above,

$$
P_{\nu}^{K}\left(\frac{d \nu \phi}{d \nu}\right)=\frac{E_{\mu}^{K}(h)}{h \circ T^{K}} P_{\mu}^{K}\left(\frac{d \mu \phi}{d \mu}\right)=\frac{P_{\mu}^{K}(h)}{h} \frac{h}{h \circ T^{K}} .
$$

Then apply Proposition 7.2. 
4. Recurrence of $\nu$ is equivalent to recurrence of $\left[P_{\nu}^{K}(d \nu \phi / d \nu)\right]^{-1}=\omega_{\nu}$ [11], and recurrence of a cocycle is invariant under cohomology.

Corollary 7.5. Under the hypotheses of Proposition 7.4, the following are equivalent:

(1) $T$ admits a $\sigma$-finite measure $\nu \sim \mu$.

(2) $P_{\mu}^{K}(d \mu \phi / d \mu)$ is a conditional coboundary for $T^{K}$ for all $\phi \leq K$ (cf. Definition 4.3).

(3) For some $\phi \in\left[S_{T}^{1}\right], P_{\mu}^{1}(d \mu \phi / d \mu)$ is a conditional coboundary for $T$.

Proof. (2) $\Rightarrow(1)$ If $P_{\mu}^{K}(d \mu \phi / d \mu)=\left(h \circ T^{K}\right) / E_{\mu}^{K}(h)=\left[\omega_{\mu}^{K}(x)\right]^{-1}$ a.e., then for $\nu=h d \mu$,

$$
\begin{aligned}
P_{\nu}^{K}\left(\frac{d \nu \phi}{d \nu}\right) & =\left[\omega_{\nu}^{K}(x)\right]^{-1}=\left[\omega_{\mu}^{K}(x)\right]^{-1} \frac{E_{\mu}^{K}(h)}{h \circ T^{K}} \quad \text { (by Lemma 3.5) } \\
& =\frac{h \circ T^{K}}{E_{\mu}^{K}(h)} \frac{E_{\mu}^{K}(h)}{h \circ T^{K}}=1
\end{aligned}
$$

so $\nu$ is invariant by Proposition 7.3.

(1) $\Rightarrow(2)$ If $T$ admits an invariant measure $\nu \sim \mu$, then

$$
1=P_{\nu}^{K}\left(\frac{d \nu \phi}{d \nu}\right)=\left[\omega_{\nu}^{K}(x)\right]^{-1}=\left[\omega_{\nu}^{K}(x)\right]^{-1} \frac{E_{\mu}^{K}(h)}{h \circ T^{K}}=P_{\mu}^{K}\left(\frac{d \mu \phi}{d \mu}\right) \frac{E_{\mu}^{K}(h)}{h \circ T^{K}}
$$

so the result follows.

$(2) \Rightarrow(3)$ is trivial.

(3) $\Rightarrow$ (1) follows from 7.1(1) and Theorem 4.6.

The question of a good notion of orbit equivalence for endomorphisms depends upon a better understanding of the cocycle $h_{*}=h / E_{\mu}^{1}(h)$ and the related Markovian $h_{*} \omega_{\mu}=h / P_{\mu}^{1}(h)$. For any $T$ one can find measurable, even integrable $h$ on $X$ for which the above cocycles are transient; this is a consequence of a construction of Eigen and Silva [7]. We pose three basic questions:

(1) Given any ergodic conservative nonsingular endomorphism $T$ which is noninvertible, is there always an equivalent recurrent measure? By Proposition 5.4 the answer is yes if and only if there exists a positive measurable function $h$ such that the cocycle $h / P_{\mu}^{1}(h)$ is recurrent for $T$.

(2) If $\nu$ and $\mu$ are equivalent recurrent measures for $T$, are they cohomologous?

(3) Obviously the $T^{-1} \mathscr{B}$-measurability of $h$ is a sufficient condition for the cocycle $h_{*}$ to be recurrent and the measures $\mu$ and $h d \mu$ to be cohomologous. Is it necessary? If $T$ admits an equivalent invariant measure, the answer is yes [24].

We conclude by mentioning some interesting topics in endomorphisms related to this study. The idea of computing the quotient relation of $R_{T}$ by the relation $S_{T}$ is a natural one and has been studied by Schmidt [20], as well as 
Dajani and the author, and others (see [6] for references). Invertible extensions, and the connections with recurrent measures, have been studied by Eigen, Silva, Thieullen, Dajani and the author (see e.g., $[6,7,8,18,23,24]$ ).

\section{BIBLIOGRAPHY}

1. J. Aaronson, M. Lin, and B. Weiss, Mixing properties of * Markov operators and ergodic transformations and ergodicity of Cartesian products, Israel J. Math. 33 (1979), 198-224.

2. G. Brown and A. Dooley, Ergodic measures are of weak product type, Math. Proc. Cambridge Philos. Soc. 98 (1985), 129-145.

3. R. Butler and K. Schmidt, An information cocycle for groups of non-singular transformations, Z. Wahrsch. Verw. Gebiete 69 (1985), 347-360.

4. A. Connes, J. Feldman, and B. Weiss, An amenable equivalence relation is generated by a single transformation, Ergodic Theory Dynamical Systems 1 (1981), 431-450.

5. I. Cornfeld, S. Fomin, and Y. Sinai, Ergodic theory, Springer-Verlag, Berlin and New York, 1982.

6. K. Dajani and J. Hawkins, Rohlin factors, product factors, and joinings for $n$-to-one maps, Indiana Univ. Math. J. 42 (1993), 237-258.

7. S. Eigen and C. Silva, $A$ structure theorem for $n$-to-one endomorphisms and existence of non-recurrent measures, J. London Math. Soc. (2) 40 (1989), 441-451.

8. T. Hamachi, On a Bernoulli shift with non-identical factor measures, Ergodic Theory Dynamical Systems 1 (1981), 273-284.

9. T. Hamachi and M. Osikawa, Ergodic groups of automorphisms of Krieger's theorems, Sem. on Math. Sci., Keio Univ., 1981.

10. J. Hawkins, Ratio sets of endomorphisms which preserve a probability measure, Measure and Measurable Dynamics, Contemp. Math., vol. 40, Amer. Math. Soc., Providence, RI, 1989, pp. $159-170$.

11. J. Hawkins and C. Silva, Remarks on recurrence and orbit equivalence of nonsingular endomorphisms, Lecture Notes in Math., vol. 1342, Springer-Verlag, Berlin and New York.

12. __ Noninvertible transformations admitting no absolutely continuous $\sigma$-finite invariant measure, Proc. Amer. Math. Soc. 111 (1989), 455-463.

13. S. Kakutani, On equivalence of infinite product measures, Ann. of Math. (2) 49 (1948), 214-224.

14. W. Krieger, On ergodic flows and isomorphism of factors, Math. Ann. 223 (1976), 19-70.

15. R. Mañe, Ergodic theory and differentiable dynamics, Springer-Verlag, Berlin and New York, 1987.

16. W. Parry, Entropy and generators in ergodic theory, Benjamin, New York, 1969.

17. V. Rohlin, On the fundamental ideas of measure theory, Amer. Math. Soc. Transl. 71 (1952), $1-54$.

18. __ Exact endomorphisms of a Lebesgue space, Amer. Math. Soc. Transl. Ser. 239 (1964), 1-36.

19. K. Schmidt, Cocycles of ergodic transformation groups, New Delhi, India, 1977.

20. _ Strong ergodicity and quotients of equivalence relations, Proc. Centre Math. Anal. Austral. Nat. Univ. 16 (1988), 300-311.

21. —_, Algebraic ideas in ergodic theory, CBMS Regional Conf. Ser. in Math., vol. 76, Amer. Math. Soc., Providence, RI, 1990.

22. F. Schweiger, Number theoretical endomorphisms with $\sigma$-finite invariant measure, Israel $\mathbf{J}$. Math. 21 (1975), 308-318.

23. C. Silva, On $\mu$-recurrent nonsingular endomorphisms, Israel J. Math 61 (1988), 1-13.

24. C. Silva and P. Thieullen, The subadditive ergodic theorem and recurrence properties of Markovian transformations, J. Math. Anal. Appl. (to appear). 
25. P. Walters, Some results on the classification of non-invertible measure preserving transformations, Recent Advances in Topological Dynamics, Lecture Notes in Math., vol 318, Springer-Verlag, Berlin and New York, 1973, pp. 266-276.

26. U. Krengel, Transformations without finite invariant measure have strong generators, Lecture Notes in Math., vol. 160, Springer-Verlag, Berlin, 1970, pp. 135-157.

Department of Mathematics, University of North Carolina at Chapel Hill, Chapel HiLl, North Carolina 27599

E-mail address: jmh@math.unc.edu 